\title{
Evolução da resistência mecânica e dos produtos de hidratação de um cimento de aluminato de cálcio, visando sua aplicação em endodontia
}

\section{(Mechanical strength and hydrate products evolution of calcium aluminate cement for endodontic applications)}

\author{
A.P. Luz, N.Z. Borba, V. C. Pandolfelli \\ Departamento de Engenharia de Materiais, Universidade Federal de S. Carlos - UFSCar \\ Rod. Washington Luiz, km 235, C. P. 676, S. Carlos, SP, 13565-905 \\ anapaula.light@gmail.com,vicpando@power.ufscar.br
}

\begin{abstract}
Resumo
Atualmente o agregado trióxido mineral (MTA) é o cimento mais utilizado para retrobturação em endodontia. Porém, apesar de sua composição ser semelhante a do cimento Portland convencional, seu elevado custo, longo tempo de endurecimento e baixa resistência mecânica tem motivado a busca por materiais alternativos para esta aplicação. Baseado nisto, neste trabalho foi avaliado a evolução da resistência mecânica e das fases cristalinas durante a hidratação de um cimento de aluminato de cálcio (CAC), visando seu uso em tratamentos endodônticos. Para isto, amostras do cimento Secar 71 foram preparadas e mantidas em contato com água ou SBF (simulated body fluid) ao longo de 15 dias a $37^{\circ} \mathrm{C}$. Ensaios de compressão uniaxial, porosidade aparente, difração de raios $\mathrm{X}$ e termogravimetria foram utilizados na avaliação dos corpos de prova após 1, 3, 7 e 15 dias de cura. Verificou-se que as principais fases formadas foram $\mathrm{CAH}_{10}, \mathrm{C}_{2} \mathrm{AH}_{8}, \mathrm{C}_{3} \mathrm{AH}_{6}$ e $\mathrm{AH}_{3}$. Quando em presença de SBF, devido ao seu efeito retardador algumas mudanças na quantidade dos produtos de hidratação do CAC foram observadas, causando efeitos na resistência mecânica do mesmo. Além disso, o presente estudo aponta que a antecipação ou retardamento da formação de algumas fases pode induzir alterações nas propriedades dos cimentos.
\end{abstract}

Palavras-chave: cimento de aluminato de cálcio (CAC), hidratação, resistência mecânica, endodontia.

\begin{abstract}
Mineral trioxide aggregate (MTA) is the most used retrograde filling cement in the endodontic area. Nevertheless, although its composition is similar to the conventional Portland cement, its high cost, long setting time and low mechanical strength have led to a continuous search for new alternative materials. Considering these aspects, the mechanical strength and crystalline phase evolution of calcium aluminate cement $(C A C)$, during its hydration process, have been evaluated in this work aiming to apply such material for endodontic treatments. Secar 71 cement samples were prepared and kept in contact with water or SBF (simulated body fluid) for 15 days at $37^{\circ} \mathrm{C}$. Compressive strength, apparent porosity, $X$ ray diffraction and thermogravimetric tests were carried out for the samples evaluation after 1, 3, 7 and 15 days. The main identified phases were $C A H_{10}, C_{2} A H_{8}, C_{3} A H_{6}$ and $A H_{3}$. When in the presence of $S B F$, due to its delaying effect, some changes in the amount of the hydrates in the CAC samples were observed, which affected the mechanical behavior of the cement. Additionally, this study pointed out that the earliness or delaying formation of some phases can induce changes in the cement properties.
\end{abstract}

Keywords: calcium aluminate cement (CAC), hydration, mechanical strength, endodontic.

\section{INTRODUÇÃO}

A endodontia é a especialidade da odontologia responsável pelo estudo da polpa dentária de todo o sistema de canais radiculares e dos tecidos periapicais, bem como das doenças que os afligem. Uma das principais finalidades da terapia endodôntica é a anti-sepsia e eliminação de agentes irritantes como bactérias e restos necróticos presentes no interior do sistema de canais radiculares, seguida da obturação deste sistema da forma mais hermética possível (Fig. 1) [1].

Os materiais convencionalmente utilizados como obturadores no tratamento endodôntico são: a gutapercha, amalgama, cimentos resinosos, os cimentos à base de óxido de zinco e eugenol, e os cimentos contendo hidróxido de cálcio. Porém, nos anos 90 o MTA (Agregado de Trióxido Mineral) foi apresentado com a finalidade de ser utilizado como um novo material reparador. $\mathrm{O}$ cimento MTA é constituído principalmente por óxidos de cálcio, de alumínio e de ferro (entre outros em menor quantidade), dióxido de silício, sulfato de cálcio e óxido de bismuto, cuja finalidade deste último é conferir ao material algum grau de radiopacidade [2].

Ao longo dos últimos anos vários estudos foram realizados para a avaliação das propriedades físico-químicas do MTA [3-5]. Nestes trabalhos foi evidenciado que as principais vantagens de sua aplicação são [6, 7]: (1) menor 


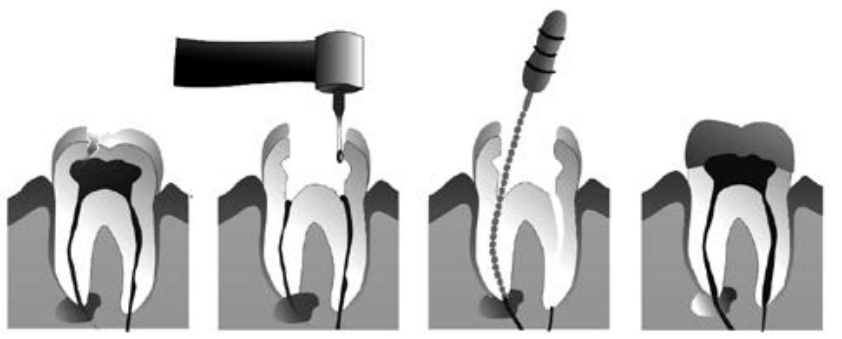

Figura 1: Esboço simplificado das etapas de um tratamento endodôntico [1].

[Figure 1: Simplified steps sketch for an endodontic treatment [1].]

microinfiltração, (2) biocompatibilidade com indução da produção de citocinas e osteocalcina, permitindo a aderência das células ao material, e (3) capacidade antibacteriana. Por outro lado, clinicamente este cimento ainda apresenta dificuldades durante sua manipulação e inserção à cavidade retrógrada. Adicionalmente, seu tempo de pega é considerado muito longo, possui baixa resistência mecânica à compressão $(<30 \mathrm{MPa})$ e sua especificidade eleva seu preço de mercado, inviabilizando seu uso rotineiro para a maioria da população [8]. Em virtude desses fatores negativos, no momento há uma busca contínua para o desenvolvimento de um material que se aproxime ao máximo de todas as propriedades almejadas a essa finalidade. Estudos recentes atestaram a capacidade do cimento de aluminato de cálcio (CAC) em prevenir a microinfiltração da bactéria Enterococcus faecalis quando este foi aplicado como retrobturador de canais radiculares em um estudo in vitro, evidenciando sua característica de propiciar a biocompatibilidade [9]. Além disso, cimentos aluminosos vêm sendo utilizados na reparação de defeitos ósseos, já que sua composição e seu coeficiente de expansão são bastante semelhantes ao do dente e osso humano [10,11].

Até o momento são poucos os trabalhos encontrados na literatura que avaliam a resistência mecânica de cimentos de aluminato de cálcio, quando estes são expostos as condições existentes em tratamentos endodônticos (em contato com fluidos corporais e a temperatura de $37^{\circ} \mathrm{C}$ ) $[6$, 11]. Com este intuito, este trabalho aborda a investigação das propriedades mecânicas e da evolução das fases geradas durante a hidratação de um CAC. As amostras obtidas após imersão em água destilada ou SBF (simulated body fluid) foram analisadas por meio das técnicas de compressão uniaxial, porosidade aparente, difração de raios X (DRX) e termogravimetria (TG). Atualmente, o CACé um biomaterial bastante promissor e, por meio de novos estudos, espera-se aperfeiçoar e expandir seu uso como um material reparador no campo da endodontia.

\section{MATERIAIS E MÉTODOS}

O cimento Secar 71 (Kerneos, França - Tabela I) foi preparado para as avaliações deste trabalho utilizandose a razão água/cimento $(\mathrm{A} / \mathrm{C})$ de 0,3 . Corpos de prova cilíndricos $(\mathrm{D}=11 \mathrm{~mm} \times \mathrm{H}=22 \mathrm{~mm})$ foram moldados sob leve vibração e mantidos em ambiente úmido (umidade relativa $\sim 100 \%$ ) durante $20 \mathrm{~h}$.
Tabela I - Composição química do cimento Secar 71. [Table I - Chemical composition of Secar 71.]

\begin{tabular}{cc}
\hline Composição Química & Teor (\%-peso) \\
\hline $\mathrm{CaAl}_{2} \mathrm{O}_{4}$ (ou CA) & 56 \\
$\mathrm{CaAl}_{4} \mathrm{O}_{7}($ ou CA $)$ & 40 \\
$\mathrm{Al}_{2} \mathrm{O}_{3}$ & 2 \\
$\mathrm{Ca}_{12} \mathrm{Al}_{14} \mathrm{O}_{33}\left(\right.$ ou C $\left._{12} \mathrm{~A}_{7}\right)$ & 2 \\
\hline
\end{tabular}

Após este período, as amostras foram desmoldadas, mergulhadas em recipiente contendo $\mathrm{H}_{2} \mathrm{O}$ destilada ou SBF (Tabela II) e armazenadas dentro de uma estufa (Nova Ética, modelo $403 / \mathrm{D}$ ) a $37{ }^{\circ} \mathrm{C} \pm 1{ }^{\circ} \mathrm{C}$. Ao completar $1,3,7$ e 15 dias de cura, cerca de 5 amostras do cimento hidratado foram submetidas a ensaios de compressão uniaxial (procedimento baseado na norma ISO 9917-1) em uma máquina de ensaios mecânicos MTS $810 \mathrm{com}$ taxa de deslocamento de $0,5 \mathrm{~mm} / \mathrm{min}$ e célula de carga de $50 \mathrm{kN}$. Os corpos de prova recolhidos após o ensaio mecânico foram macerados e submetidos a secagem em forno microondas (Brastemp, modelo BMS25), usando a potência máxima deste equipamento durante o tempo de 6 min (foi verificado previamente que nesta condição houve a remoção da água livre do cimento, obtendo-se um valor constante para a massa do material analisado).

Após a interrupção da hidratação os cimentos ainda foram comínuidos $(\mathrm{dp}<45 \mu \mathrm{m})$ usando equipamento AMEF (modelo AMP1-M) e analisados por meio das técnicas de DRX (equipamento Bruker, modelo D8 focus, com radiação $\mathrm{CuK} \alpha[\lambda=1,5418 \AA]$ e filtro de níquel, empregandose $40 \mathrm{~mA}, 40 \mathrm{mV}$ e passo $=0,02$ ) e $\mathrm{TG}$ (equipamento NETZSCH STA 449, taxa de aquecimento de $10{ }^{\circ} \mathrm{C} / \mathrm{min}$ em fluxo de ar sintético $\left(80 \% \mathrm{~N}_{2}-20 \% \mathrm{O}_{2}\right)$ de $50 \mathrm{~cm}^{3} / \mathrm{min}$ e usando $\alpha-\mathrm{Al}_{2} \mathrm{O}_{3}$ como padrão de correção). A partir das curvas de TG e DTG, os teores de alguns hidratos ainda

Tabela II - Reagentes e suas respectivas quantidades utilizadas na preparação da solução SBF (1000 mL).

[Table II - Reagents and their respective amounts for preparation of simulated body fluid - SBF $(1000 \mathrm{~mL})$.]

\begin{tabular}{cc}
\hline Reagentes & Quantidades \\
\hline Água destilada & $750 \mathrm{~mL}$ \\
$\mathrm{NaCl}$ & $7,996 \mathrm{~g}$ \\
$\mathrm{NaHCO}_{3}$ & $0,350 \mathrm{~g}$ \\
$\mathrm{KCl}$ & $0,224 \mathrm{~g}$ \\
$\mathrm{~K}_{2} \mathrm{HPO}_{4}$ & $0,174 \mathrm{~g}$ \\
$\mathrm{MgCl}_{2} \cdot 6 \mathrm{H}_{2} \mathrm{O}$ & $0,305 \mathrm{~g}$ \\
$\mathrm{HCl} 1 \mathrm{~mol}^{-\mathrm{L}}$ & $40 \mathrm{~mL}$ \\
$\mathrm{CaCl}_{2}$ & $0,278 \mathrm{~g}$ \\
$\mathrm{Na}_{2} \mathrm{SO}_{4}$ & $0,071 \mathrm{~g}$ \\
$\left(\mathrm{CH}_{2} \mathrm{OH}_{2}\right) \mathrm{CNH}_{2}$ & $6,057 \mathrm{~g}$ \\
$\mathrm{HCl} \mathrm{l} \mathrm{mol}_{2} \mathrm{~L}$ & quantidade apropriada para \\
& ajuste do pH em 7,25 \\
\hline
\end{tabular}


foram estimados utilizando-se o procedimento descrito no trabalho de Dweck et al. [12], o qual considera as perdas de massa das curvas de TG entre as temperaturas inicial e final dos picos obtidos por DTG e compara tais informações com as reações de decomposição das fases e as perdas de massa teóricas previstas pela estequiometria. Os resultados de DRX também foram avaliados por meio do método de Rietveld (programa Topas 4.2), sendo estimada a quantidade dos hidratos contidos nas amostras coletadas nos diversos intervalos de tempo escolhidos. Devido a ausência de informações detalhadas sobre a estrutura e simetria das fases $\mathrm{CAH}_{10}$ e $\mathrm{C}_{2} \mathrm{AH}_{8}$, optou-se por efetuar uma avaliação semi-quantitativa destes hidratos (programa EVA). Para isto foi realizada a medida da área dos picos mais intensos dos hidratos $\mathrm{CAH}_{10}$ e $\mathrm{C}_{2} \mathrm{AH}_{8}$, seguida pela comparação destes valores com as áreas dos picos dos hidratos $\mathrm{C}_{3} \mathrm{AH}_{6}$ e $\mathrm{Al}(\mathrm{OH})_{3}$ e normalização dos dados para posterior análise. Além disso, a medida da porosidade aparente das amostras do CAC (antes da interrupção da hidratação) foi realizada usando o método de Archimedes (ASTM C380-00), sendo empregado querosene como meio líquido.

\section{RESULTADOS E DISCUSSÃO}

A propriedade mecânica mais amplamente utilizada no estudo de cimentos é a resistência à compressão. Neste tipo de ensaio a resistência do corpo de prova irá variar de acordo com a distribuição de tamanhos e a localização dos poros no material [10]. Portanto, é muito importante minimizar o tamanho e a quantidade de poros no material para alcançar elevada resistência e durabilidade. Um número elevado de parâmetros influenciam a porosidade, sendo os dois mais importantes a razão água/cimento e o ar aprisionado na pasta cimentícia. $\mathrm{O}$ valor de $\mathrm{A} / \mathrm{C}=0,3$ utilizado neste trabalho e a prática de moldagem sob vibração tendem a favorecer a obtenção de corpos de prova com menor porosidade e elevada resistência mecânica, como apresentado na Fig. 2.

Naturalmente a hidratação do cimento aluminoso consiste em um processo dinâmico onde após o contato inicial com a água de mistura verifica-se: (1) a hidroxilação da superfície das partículas do cimento, seguida pela dissolução e liberação dos íons $\mathrm{Ca}^{2+}, \mathrm{Al}(\mathrm{OH})_{4}^{-}$e $\mathrm{OH}^{-}$, (2) formação de uma pequena quantidade de hidratos em forma de gel, se a concentração da solução atingir o limite de supersaturação dos hidratos $\mathrm{C}_{2} \mathrm{AH}_{8}$ e $\mathrm{AH}_{3}$ (onde $\mathrm{C}=\mathrm{CaO}, \mathrm{A}=\mathrm{Al}_{2} \mathrm{O}_{3}$ e $\mathrm{H}=\mathrm{H}_{2} \mathrm{O}$ ), (3) dissolução do sólido até ser atingido o ponto de saturação, (4) período de indução onde os núcleos formados atingirão
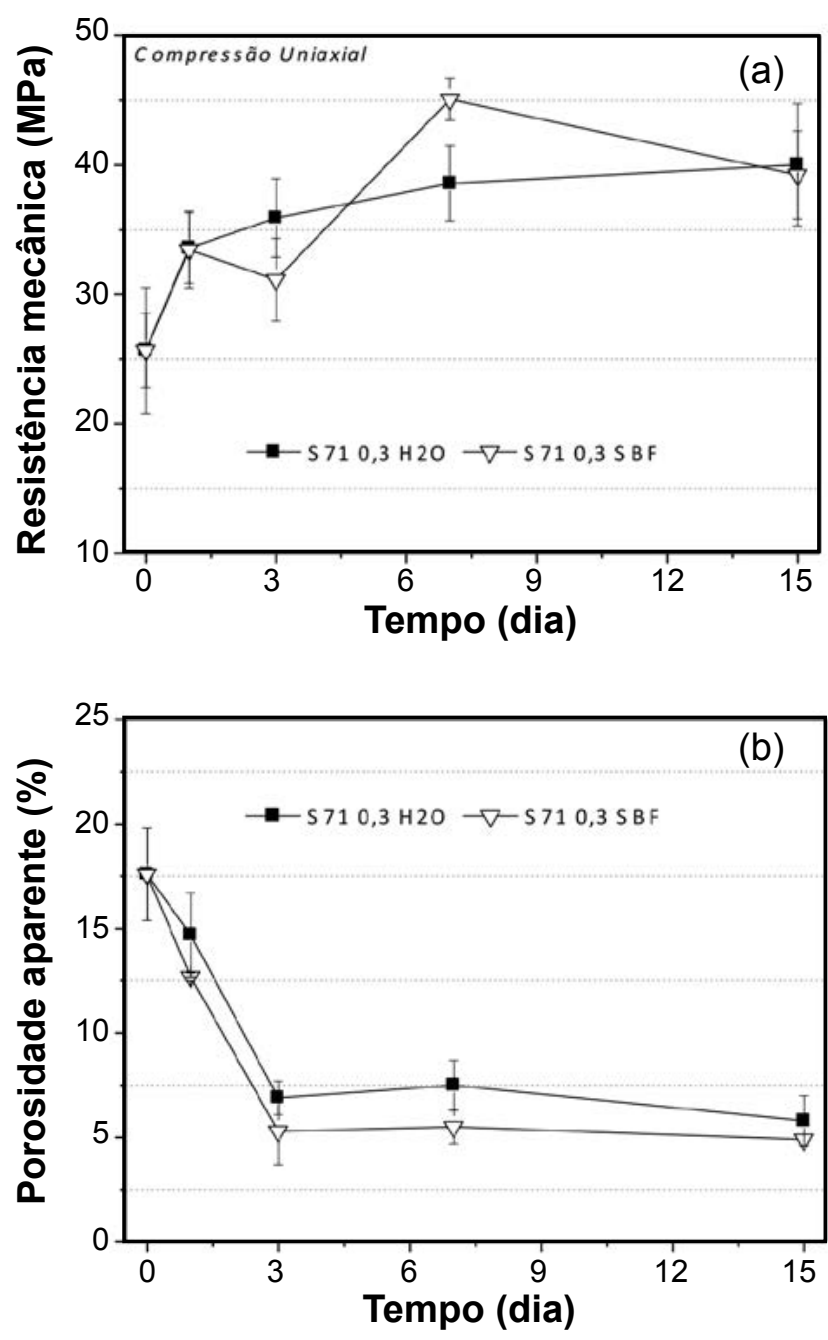

Figura 2: Resultados de compressão uniaxial e porosidade aparente das amostras do cimento Secar 71 mantidas mergulhadas em $\mathrm{H}_{2} \mathrm{O}$ ou SBF a $37^{\circ} \mathrm{C}$.

[Figure 2: Compressive strength and apparent porosity results of Secar 71 samples kept in $\mathrm{H}_{2} \mathrm{O}$ or SBF at $37^{\circ} \mathrm{C}$.]

Tabela III - Características dos principais hidratos que podem ser formados na estrutura dos cimentos de aluminato de cálcio [10].

[Table III - Features of the main hydrates that can be formed in the calcium aluminate cement structure [10].]

\begin{tabular}{ccccccc}
\hline \multirow{2}{*}{ Fases } & \multicolumn{2}{c}{ Composição Química $(\%-\mathrm{p})$} & Temperatura & Estrutura & Cristalina & $\begin{array}{c}\text { Densidade } \\
\left(\mathrm{g} / \mathrm{cm}^{3}\right)\end{array}$ \\
& $\mathrm{CaO}$ & $\mathrm{Al}_{2} \mathrm{O}_{3}$ & $\mathrm{H}_{2} \mathrm{O}$ & $\left({ }^{\circ} \mathrm{C}\right)$ & Hexagonal & 1,72 \\
$\mathrm{CAH}_{10}$ & 16,6 & 30,1 & 53,5 & $<20$ & Hexagonal & 1,95 \\
$\mathrm{C}_{2} \mathrm{AH}$ & 31,3 & 28,4 & 40,3 & $20-30$ & Cúbica & 2,52 \\
$\mathrm{C}_{3} \mathrm{AH}$ & 44,4 & 27,0 & 28,6 & $>30$ & Hexagonal & 2,42 \\
$\mathrm{AH}_{3}$ & - & 65,4 & 34,6 & $>30$ & \\
\hline
\end{tabular}


tamanho e quantidade críticos, (5) precipitação massiva dos hidratos, levando a queda na concentração dos íons da solução e retorno do processo para a situação encontrada na etapa (1) [13].

Pensando somente no aspecto físico, o crescimento dos hidratos e o intertravamento e ligação entre as fases promove o aumento da resistência mecânica [11, 13]. Além disso, devido ao reduzido teor de água utilizado na etapa de mistura e moldagem das amostras, ainda haverá uma quantidade significativa de fases anidras contidas no cimento, que em contato com a água ou SBF (líquidos utilizados no armazenamento dos corpos de prova durante a cura), estas poderão reagir e dar continuidade ao processo de hidratação, resultando no aumento contínuo da resistência mecânica e redução da porosidade entre 1-15 dias (Fig. 2). Por outro
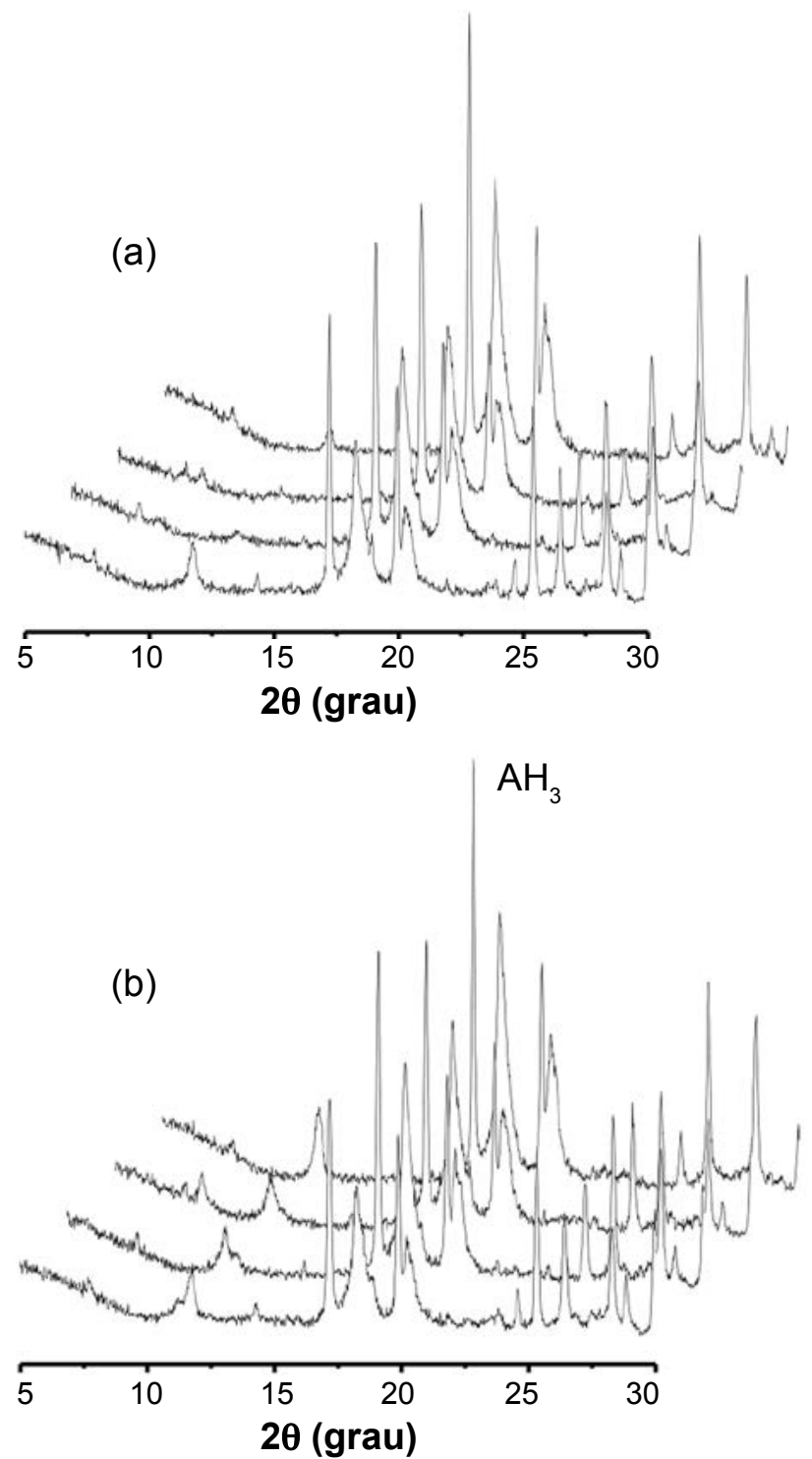

Figura 3: Difratogramas de raios $\mathrm{X}$ das amostras do cimento Secar 71 preparadas com $\mathrm{A} / \mathrm{C}=0,3$ e mantidas a $37^{\circ} \mathrm{C}$ em contato com (a) água destilada ou (b) SBF.

[Figure 3: XRD profiles of Secar 71 samples prepared with $W / C=$ 0.3 and kept at $37^{\circ} \mathrm{C}$ in (a) distilled water or (b) SBF.] lado, a temperatura a que o cimento é exposto modifica significativamente os hidratos resultantes (Tabela III) devido a mudança em suas solubilidades, sendo que a $37{ }^{\circ} \mathrm{C}$ (temperatura corporal) há o favorecimento da precipitação das fases $\mathrm{C}_{3} \mathrm{AH}_{6}$ e $\mathrm{AH}_{3}$ no cimento Secar 71 (Fig. 3).

Observou-se que, quando em presença de SBF houve a queda na resistência mecânica do cimento no $3^{\circ}$ dia, sendo esta seguida por um aumento significativo (valor máximo $=$ 45,7 $\mathrm{MPa}$ ) até o $7^{\circ}$ dia de cura. Vale destacar também que a reprodutibilidade destes dados foi confirmada por meio da realização de ensaios mecânicos adicionais. No entanto, após 15 dias as amostras mantidas em $\mathrm{H}_{2} \mathrm{O}$ destilada ou SBF apresentaram resultados muito similares para as duas propriedades avaliadas - resistência mecânica e porosidade aparente.

As mudanças no comportamento do Secar 71 quando em contato com o SBF podem estar relacionadas a presença dos íons $\mathrm{PO}_{4}^{3-}, \mathrm{HPO}_{4}^{2-}$ e $\mathrm{CO}_{3}^{2-}$ que alteram o equilíbrio e induzem algumas mudanças na composição dos produtos finais, especialmente na superfície dos corpos de prova. Alguns trabalhos sugerem que baseados na solubilidade e $\mathrm{pH}$ desenvolvidos no meio líquido, a mistura das fases $\mathrm{Al}(\mathrm{OH})_{3}$ e $_{3} \mathrm{AH}_{6}$ podem ser convertidas em $\mathrm{Al}(\mathrm{OH})_{3}$ e apatita quando em contato com SBF (Eq. A) $[2,10]$.

$$
\begin{aligned}
& \mathrm{Ca}_{3}\left[\mathrm{Al}(\mathrm{OH})_{4}\right]_{2}(\mathrm{OH})_{4}+2 \mathrm{Ca}^{2+}+\mathrm{HPO}^{2-}{ }_{4} 2 \mathrm{H}_{2} \mathrm{PO}_{4}^{-} \rightarrow \\
& \mathrm{Ca}_{5}\left(\mathrm{PO}_{4}\right)_{3}(\mathrm{OH})+2 \mathrm{Al}(\mathrm{OH})_{3}+5 \mathrm{H}_{2} \mathrm{O}
\end{aligned}
$$

Além disso, a presença dos íons $\mathrm{CO}_{3}^{2-}$ também pode promover a precipitação de $\mathrm{CaCO}_{3}$, quando em um ambiente com elevado $\mathrm{pH}$. Assim, quando o CAC é colocado em contato com soluções corporais, acredita-se que uma zona interfacial contendo uma mistura de diversos produtos precipitados será criada próxima ao local de contato com o líquido. Por outro lado, ao mover-se para o interior da amostra, $\mathrm{C}_{3} \mathrm{AH}_{6}$ e $\mathrm{Al}(\mathrm{OH})_{3}$ são as fases dominantes, como verificado nos resultados de DRX (Fig. 3b).

Acredita-se que não foi possível identificar a presença das fases $\mathrm{Ca}_{3}\left(\mathrm{PO}_{4}\right)_{3}(\mathrm{OH})$ e $\mathrm{CaCO}_{3}$ nas amostras do cimento Secar 71, por estas não se encontrarem em suas formas cristalinas ou seus teores estarem abaixo do limite de detecção da técnica de DRX $( \pm 5 \%)$. Novas investigações ainda são necessárias para compreender e comprovar se tais transformações podem afetar o comportamento mecânico do cimento ao longo dos 15 dias de cura.

De acordo com os resultados de DRX (qualitativo - Fig. $3 \mathrm{e}$ quantitativo - Tabela IV), os principais hidratos identificados foram $\mathrm{C}_{3} \mathrm{AH}_{6} \mathrm{e} \mathrm{AH}_{3}$, porém outros compostos como $\mathrm{CAH}_{10}$ e $\mathrm{C}_{2} \mathrm{AH}_{8}$ estavam presentes em menores quantidades nas condições avaliadas (água ou SBF). Sabe-se que o limite de detecção desta técnica é de $\pm 5 \%$ e que, apesar de terem sido identificados, os picos do hidrato $\mathrm{C}_{2} \mathrm{AH}_{8}$ apresentaram reduzidos valores de intensidade e área quando comparados com as demais fases presentes. Além disso, os cálculos para a determinação dos teores das fases (análise quantitativa) realizados no programa Topas 4.2 apresentam um desvio médio de $\pm 1 \%$ e, por sua vez, os valores mostrados na 
Tabela IV - Comparação entre os resultados de DRX quantitativo das amostras do cimento Secar $71(\mathrm{~A} / \mathrm{C}=0,3)$ mantidas em $\mathrm{H}_{2} \mathrm{O}$ ou $\mathrm{SBF}$ a $37^{\circ} \mathrm{C}$.

[Table IV - Comparison of the quantitative XRD results of Secar 71 samples $(W / C=0.3)$ kept in $\mathrm{H}_{2} \mathrm{O}$ or $\mathrm{SBF}$ at $37^{\circ} \mathrm{C}$.]

\begin{tabular}{cccccccc}
\hline \multirow{2}{*}{ Amostras } & \multirow{6}{c}{ Tempo de cura (dias) } & $\mathrm{CA}$ & $\mathrm{CA}_{2}$ & $\mathrm{CAH}_{10}$ & $\mathrm{C}_{2} \mathrm{AH}_{8}$ & $\mathrm{C}_{3} \mathrm{AH}_{6}$ & $\mathrm{Al}(\mathrm{OH})_{3}$ \\
\hline \multirow{3}{*}{$S 71-\mathrm{H}_{2} \mathrm{O}$} & 1 & 8,5 & 16,3 & 8,3 & 0,5 & 27,2 & 39,2 \\
& 3 & 6,1 & 9,1 & 0 & 0,9 & 33,4 & 49,8 \\
& 7 & 4,1 & 5,7 & 0 & 0,8 & 36,9 & 52,2 \\
& 15 & 2,4 & 2,9 & 3,0 & 0,6 & 38,2 & 52,8 \\
\multirow{2}{*}{$S 71-S B F$} & 1 & 9,9 & 18,3 & 9,0 & 1,5 & 26,6 & 34,5 \\
& 3 & 6,7 & 8,8 & 7,9 & 1,6 & 29,1 & 45,1 \\
& 7 & 4,6 & 5,3 & 8,5 & 2,9 & 30,3 & 48,1 \\
& 15 & 3,0 & 3,8 & 9,2 & 1,0 & 33,2 & 49,8 \\
\hline \multirow{2}{*}{$S$} & 15 & & & & & &
\end{tabular}
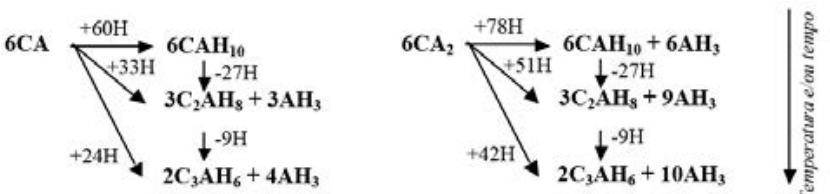

Figura 4: Equações que representam as reações de formação dos hidratos em cimentos de aluminato de cálcio [14].

[Figure 4: Representative equations for the hydrates generation reactions in calcium aluminate cements [14].]

Tabela IV para o $\mathrm{C}_{2} \mathrm{AH}_{8}$ (entre $0,5-2,9 \%$-p) encontramse próximos da faixa de erro deste procedimento. Contudo, apesar destas observações, optou-se por apresentar aqui tais informações sobre este hidrato, uma vez que seus picos são detectados nos difratogramas da Fig. 3.

Quando em presença de $\mathrm{H}_{2} \mathrm{O}, \mathrm{CAH}_{10}$ somente foi identificado no $1^{\circ}$ e $15^{\circ}$ dia de cura. Este fato indica que a ausência deste hidrato no $3^{\circ}$ e $7^{\circ}$ pode estar relacionada a conversão desta fase, dando origem à $\mathrm{C}_{3} \mathrm{AH}_{6}$ e $\mathrm{Al}(\mathrm{OH})_{3}$ que são mais estáveis. Já no $15^{\circ}$ dia o re-aparecimento do $\mathrm{CAH}_{10}$ deve ser ocasionado pela hidratação das fases anidras que ainda se encontravam presentes nas amostras ou pelo excesso de água contido no meio. Além disso, pequenos picos do hidrato $\mathrm{C}_{2} \mathrm{AH}_{8}$ também puderam ser encontrados nos difratogramas deste cimento, comprovando que mesmo a $37{ }^{\circ} \mathrm{C}$ (condição onde é favorecida a geração das fases $\mathrm{C}_{3} \mathrm{AH}_{6}$ e $\mathrm{AH}_{3}$ ) a formação dos hidratos menos estáveis ainda pode ocorrer devido a grande disponibilidade de água presente no ambiente de cura. O contínuo aumento dos teores das fases $\mathrm{C}_{3} \mathrm{AH}_{6}$ e $\mathrm{Al}(\mathrm{OH})_{3}$ ao longo do tempo ocorre em virtude da hidratação do $\mathrm{CA}$ e $\mathrm{CA}_{2}$ e/ou pela conversão dos hidratos menos estáveis, conforme esquema mostrado na Fig. 4. No entanto, a presença destes hidratos de maior densidade parece afetar positivamente o comportamento do CAC, promovendo o aumento da resistência mecânica das amostras entre 1-15 dias.

Algumas mudanças significativas foram observadas na evolução das fases do cimento quando em contato com SBF.

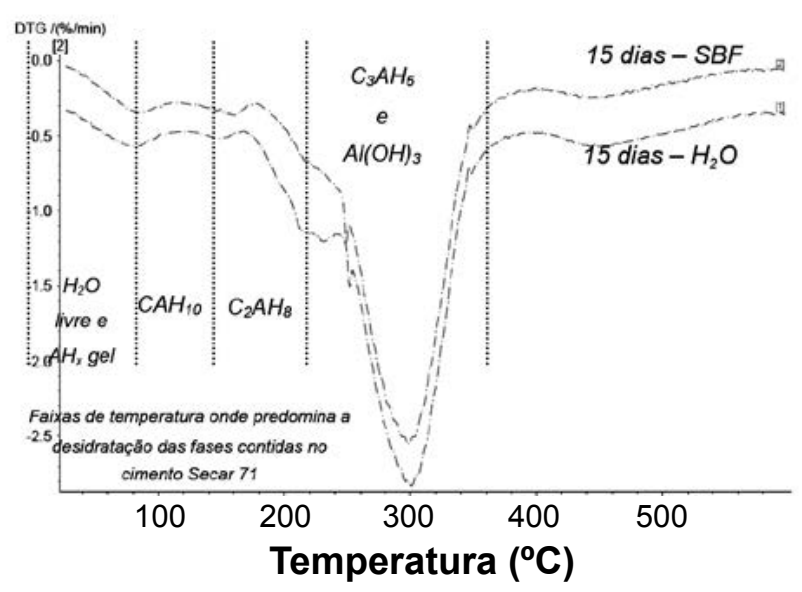

Figura 5: Curvas de DTG para as amostras do cimento Secar 71 preparadas com $\mathrm{A} / \mathrm{C}=0,3$ e mantidas em contato com $\mathrm{H}_{2} \mathrm{O}$ ou $\mathrm{SBF}$ durante 15 dias a $37^{\circ} \mathrm{C}$.

[Figure 5: DTG curves of Secar 71 samples prepared with $\mathrm{W} / \mathrm{C}=$ 0.3 and kept in $\mathrm{H}_{2} \mathrm{O}$ or SBF for 15 days at $\left.37^{\circ} \mathrm{C}.\right]$

Por exemplo, $\mathrm{CAH}_{10}$ e $\mathrm{C}_{2} \mathrm{AH}_{8}$ aparecem de maneira mais expressiva e, conseqüentemente, menores teores dos demais hidratos estão presentes nesta condição.

Uma hipótese levantada é que a presença dos íons contidos na solução SBF pode estar deslocando o equilíbrio do sistema, atuando como um retardador do processo de hidratação. Por exemplo, sais como o $\mathrm{CaCl}_{2}, \mathrm{MgCl}_{2}$ e $\mathrm{NaCl}$ (que estão presentes no SBF) são conhecidos por alterarem a sequiência das reações, retardando o tempo de pega do cimento de aluminato de cálcio quando estes são adicionados a este ligante [11]. Estas mudanças devem ser as principais responsáveis pelas alterações verificadas no comportamento do CAC quando comparado com os testes do material mantido em água.

De acordo com os resultados obtidos pela técnica de termogravimetria (curvas de DTG mostradas na Fig. 5), foi confirmada a presença das fases $\mathrm{C}_{3} \mathrm{AH}_{6} \mathrm{e}$ 
Tabela V - Análise da decomposição dos hidratos do cimento Secar $71\left(\mathrm{~A} / \mathrm{C}=0,3\right.$, após cura por 15 dias a $\left.37^{\circ} \mathrm{C}\right)$ obtida a partir das curvas de TG e DTG.

[Table $V$ - Analysis of the Secar 71 cement hydrates decomposition $\left(\mathrm{W} / \mathrm{C}=0.3\right.$, after 15 days at $\left.37^{\circ} \mathrm{C}\right)$ based on the results of the TG and DTG curves.]

\begin{tabular}{ccccc}
\hline $\begin{array}{c}\text { Método de interrupção da } \\
\text { hidratação }\end{array}$ & $\begin{array}{c}\mathrm{H}_{2} \mathrm{O} \text { do } \\
\mathrm{CAH}_{10} \\
(\%-\mathrm{p})\end{array}$ & $\begin{array}{c}\mathrm{H}_{2} \mathrm{O} \text { do } \mathrm{Al}(\mathrm{OH})_{3}+\mathrm{C}_{3} \mathrm{AH}_{6} \\
(\%-\mathrm{p})\end{array}$ & $\begin{array}{c}\mathrm{H}_{2} \mathrm{O} \text { dos outros } \\
\text { hidratos } \\
(\%-\mathrm{p})\end{array}$ & $\begin{array}{c}\text { Total perda de } \\
\text { massa a } 600{ }^{\circ} \mathrm{C} \\
(\%-\mathrm{p})\end{array}$ \\
\hline $\mathrm{H}_{2} \mathrm{O}$ & 0,9 & 22,7 & 5,4 & 29,1 \\
$\mathrm{SBF}$ & 1,7 & 21,3 & 6,7 & 29,7 \\
\hline
\end{tabular}

$\mathrm{Al}(\mathrm{OH})_{3}$ devido ao principal pico de decomposição identificado a $\sim 300{ }^{\circ} \mathrm{C}$. A temperatura característica onde ocorre a decomposição das fases $\mathrm{Al}(\mathrm{OH})_{3}$ e $\mathrm{C}_{3} \mathrm{AH}_{6}$ é mais precisamente em $285{ }^{\circ} \mathrm{C}$ e $300{ }^{\circ} \mathrm{C}$, respectivamente [11, 14], no entanto estas transformações encontram-se muito próximas e por esta razão apenas um pico principal pode ser observado, considerando a taxa de aquecimento utilizada $\left(10{ }^{\circ} \mathrm{C} / \mathrm{min}\right)$. Além disso, entre $180-240{ }^{\circ} \mathrm{C}$ também pode haver a decomposição do composto $\mathrm{C}_{2} \mathrm{AH}_{8}$. Assim, foram encontradas algumas dificuldades com o uso desta técnica para definir e isolar a contribuição de cada hidrato no aumento da taxa de perda de massa entre 180-300 ${ }^{\circ} \mathrm{C}$. Água livre e/ou $\mathrm{AH}_{\mathrm{x}}$ gel e $\mathrm{CAH}_{10}$ também foram identificadas devido a presença de picos próximos a 90 e $160{ }^{\circ} \mathrm{C}$

Baseando-se no procedimento descrito no trabalho de Dweck et al. [12] foram realizados alguns cálculos para estimar (de forma indireta, ou seja, pela perda de massa resultante da eliminação da água) as quantidades dos hidratos presentes nos cimentos, a partir das curvas de TG e DTG. A Tabela V apresenta tais resultados e, assim como nas análises dos difratogramas, a presença de $\mathrm{C}_{3} \mathrm{AH}_{6}$ e $\mathrm{Al}(\mathrm{OH})_{3}$ parece ser mais intensa nas amostras mantidas em água. A maior perda total de massa das amostras mantidas em SBF decorre da presença mais acentuada dos hidratos $\mathrm{CAH}_{10} \mathrm{e}$ $\mathrm{C}_{2} \mathrm{AH}_{8}$, uma vez que estas fases contem maiores teores de $\mathrm{H}_{2} \mathrm{O}$ em suas estruturas.

\section{CONCLUSÕES}

De acordo com esta investigação o cimento de aluminato de cálcio Secar 71 apresenta resistência à compressão acima de $30 \mathrm{MPa}$ (ou seja, melhor desempenho quando comparado ao MTA) $\log$ o ao primeiro dia de cura. Além disso, até o $15^{\circ}$ dia de cura ainda há um contínuo aumento no valor do módulo de ruptura, alcançando valores próximos a 40 MPa para as duas condições testadas, em água ou SBF. As principais fases formadas na estrutura do cimento durante a cura foram $\mathrm{C}_{3} \mathrm{AH}_{6}$ e $\mathrm{AH}_{3}$, porém pequenos teores de $\mathrm{CAH}_{10}, \mathrm{C}_{2} \mathrm{AH}_{8}$ também foram detectados. Quando em contato com SBF, algumas mudanças na quantidade dos produtos de hidratação foram observadas, e estas indicam que possivelmente os íons contidos na solução SBF atuam retardando o processo de hidratação, resultando na variação da resistência mecânica das amostras ao longo dos 15 dias de cura. Baseado nos resultados obtidos pode-se afirmar que o CAC avaliado apresenta um bom comportamento mecânico, atendendo os requisitos mínimos para ser aplicado como um material reparador para tratamentos endodônticos. Além disso, o presente estudo aponta quais as fases presentes que quando associadas à resistência mecânica, indicam rotas para induzir alterações nas propriedades dos cimentos conforme o interesse tecnológico.

\section{AGRADECIMENTOS}

Ao Conselho Nacional de Desenvolvimento Científico e Tecnológico (CNPq) pelo apoio a este trabalho.

\section{REFERÊNCIAS}

[1] M. R. Leonardo, Endodontia: tratamento de canais radiculares: princípios técnicos e biológicos, Artes Médicas, S. Paulo (2005) 21.

[2] M. Parirokh, M. Torabinejad, Mineral Trioxide Aggregate: A comprehensive literature review - Part 1: Chemical, physical, and antibacterial properties, J. Endod. 36 (2010) 16.

[3] M. Torabinejad, P. W. Smith, J. D. Kettering, T. R. Pitt Ford, Comparative investigation of marginal adaption of mineral trioxide aggregate and other commonly used rootend filling materials, J. Endod. 21 (1995) 295.

[4] M. Torabinejad, R. K. Higa, D. J. McKendry, T. R. Pitt Ford, Dye leakage of four root end filling materials effects of blood contamination, J. Endod. 20 (1994) 159.

[5] N. K. Sarkar, P. Caicedo, P. Ritwik, R. Moiseyeva, I. Kawashima, Physicochemical basis of the biologic properties of mineral trioxide aggregate, J. Endod. 31 (2005) 97.

[6] M. Torabinejad, C. U. Hong, F. McDonald, T. R. Pitt Ford, Physical and chemical properties of a new root-end filling material, J. Endod. 21 (1995) 349.

[7] A. U. Eldeniz, H. H. Hadimli, H. Ataoglu, D. Orstavik, Antibacterial effect of selected rootend filling materials, $\mathrm{J}$. Endod. 32 (2006) 345.

[8] T. H. Huang, T. H. Shion, C. T. Kao, S. J. Ding, The effect of setting accelerator on properties on mineral trioxide aggregate, J. Endod. 34 (2008) 590.

[9] V. C. Pandolfelli, I. R. OLiveira, M. Jacobovitz, H. L. Rosseto, Composição à base de cimento aluminoso para aplicação em endodontia e produto cimentício obtido, 
Patente No PI0704502-6A2 (2007).

[10] J. Loof, Calcium-aluminate as biomaterial. Synthesis, design and evaluation, Diss. Mestrado, Faculty of Science and Technology - Uppsala University (2008) 87p.

[11] J. E. Kopanda, G. MacZura, Production processes, properties, and applications for calcium aluminate cements, In L. D. Hart, Alumina Chemicals Science and Tecnology Handbook, Am. Cer. Soc. (1990) 171.

[12] J. Dweck, P. M. Buchler, A. C. V. Coelho, F. K.
Cartledge, Hydration of a Portland cement blended with calcium carbonate, Thermochimica Acta 346 (2000) 105.

[13] C. Parr, F. Simonin, B. Touzo, C. Wohrmeyer, B. Valdelievre, A. Namba, The impact of calcium aluminate cement hydration upon the properties of refractory castables, In proceedings TARJ meeting (2004) 1.

[14] P. Pena, A. H. De Aza, Cementos de aluminatos cálcicos. Constitución, características y aplicaciones, In Refractarios Monolíticos, Ed. Soc. Esp. Cerám. Y Vidrio (1999) 85.

(Rec. 10/05/2011, Ac. 03/09/2011) 